\title{
Eczema Herpeticum in a Patient With Hailey-Hailey Disease Confounded by Coexistent Psoriasis
}

\author{
Vidhi V. Shah, MD; Ryan Fischer, MD; Stephen Squires, MD; Viseslav Tonkovic-Capin, MD
}

\section{PRACTICE POINTS}

- Misdiagnosis of Hailey-Hailey disease (HHD) occurs because of a wide spectrum of presentations.

- Hailey-Hailey disease and psoriasis are thought to occasionally koebnerize (isomorphic response) to areas of trauma.

- Clinicians should remain suspicious and evaluate for herpes simplex virus infection in refractory or sudden exacerbation of HHD.

To the Editor:

Hailey-Hailey disease (HHD), also known as benign familial pemphigus, is an uncommon autosomaldominant skin disease. ${ }^{1}$ Defects in the ATPase type $2 \mathrm{C}$ member 1 gene, ATP2C1, result in abnormal intracellular epidermal adherence, and patients experience recurring blisters in skin folds. Longitudinal white streaks of the fingernails also may be present. ${ }^{1}$ The illness does not appear until puberty and is heightened by the second or third decade of life. Family history often suggests the presence of disease. ${ }^{2}$ Misdiagnosis of HHD occurs because of a wide spectrum of presentations. The presence of superimposed infections and carcinomas may both obscure and exacerbate this disease. ${ }^{2}$

Herpes simplex viruse types 1 and 2 (HSV-1 and HSV-2) are DNA viruses that cause common recurrent diseases. Usually, HSV-1 is associated with infection of the mouth and HSV-2 is associated with infection of the genitalia. ${ }^{3}$ Longitudinal cutaneous lesions manifest as grouped vesicles on an erythematous base. Tzanck smear of herpetic vesicles will reveal the presence of multinucleated giant cells. A direct fluorescent antibody technique also may be used to confirm the diagnosis. ${ }^{3}$

Erythrodermic HHD disease is a rare condition; moreover, there are only a few reported cases with coexistence of HHD and HSV in the literature. ${ }^{3-6}$ We report a rare presentation of erythrodermic HHD and coexistent psoriasis with HSV superinfection.

A 69-year-old man presented to an outpatient dermatology clinic for evaluation and treatment of a rash on the scalp, face, back, and lower legs. The patient confirmed a dandruff diagnosis on the scalp and face as well as psoriasis on the trunk and extremities for the last 45 years. He described a history of successful treatment with topical agents and UV light therapy. A family history revealed that the patient's father and 1 of 2 siblings had a similar rash and"skin problems."The patient had a medical history of thyroid cancer treated with radiation treatment and a partial thyroidectomy 35 years prior to the current presentation as well as incompletely treated chronic hepatitis $\mathrm{C}$.

A search of medical records revealed a punch biopsy from the posterior neck that demonstrated an acantholytic dyskeratosis with suprabasal acantholysis. Clinicians were unable to differentiate if it was Darier disease (DAR) or HHD. Treatment of the patient's seborrheic dermatitis and acantholytic disorder was successful at that time with ketoconazole shampoo, ketoconazole cream, desonide cream, and triamcinolone cream. The patient remained

Dr. Shah is from the University of South Florida, Tampa. Dr. Fischer is from Dermatology Associates of Kentucky, Lexington. Dr. Squires is from the Department of Dermatology and Dr. Tonkovic-Capin is from the Department of Dermatopathology, both at the Veterans Affairs Medical Center, Kansas City.

The authors report no conflict of interest.

Correspondence: Ryan Fischer, MD (RyanFischerUK@gmail.com). 
stable for 5 years before presenting again to the dermatology clinic for worsening rash despite topical therapies.

At the current presentation, physical examination at the outpatient dermatology clinic revealed few scaly, erythematous, eroded papules distributed on the midback; erythematous greasy scaling on the scalp, face, and chest; and pink scaly plaques with white-silvery scale on the anterior lower legs. Histopathology of a specimen from the right mid-back demonstrated acantholysis with suprabasal clefting, hyperkeratosis, and parakeratosis with no dyskeratotic cells identified. The pathologic differential diagnosis included primary acantholytic processes including Grover disease, DAR, HHD, and pemphigus. Pathology from the right shin demonstrated acanthosis, confluent parakeratosis with associated decreased granular cell layer and collections of neutrophils within the stratum corneum, spongiosis, and superficial dermal perivascular chronic inflammation with focal exocytosis and dilated blood vessels in the papillary dermis. The clinical and pathological diagnosis on the lower legs was consistent with psoriasis. Diagnoses of seborrheic dermatitis, psoriasis on the lower legs, and HHD vs DAR on the back and chest were made. The patient was instructed to continue ketoconazole shampoo, ketoconazole cream, and desonide for seborrheic dermatitis; fluocinonide ointment $0.05 \%$ to the lower legs for psoriasis; and triamcinolone cream and a bland moisturizer to the back and chest for HHD.

Over the ensuing months, the rash worsened with erythema and scaling affecting more than half of the body surface area. Topical corticosteroids and bland emollients resulted in minimal success. Biologics and acitretin were considered for the psoriasiform dermatitis but avoided due to the patient's medical history of thyroid cancer and chronic hepatitis $\mathrm{C}$ infection. Because the patient described prior success with UV light therapy for psoriasis, he requested light therapy. A subsequent trial of narrowband UVB light therapy initially improved some of the psoriasiform dermatitis on the trunk and extremities; however, after 4 weeks of treatment, the patient described pain in some of the skin and felt he was burned by minimal exposure to light therapy on one particular visit, which caused him to stop light therapy.

Approximately 2 weeks later, the patient presented to the emergency department stating his psoriasis was infected; he was diagnosed with psoriasis with secondary cellulitis and received intravenous vancomycin and piperacillin-tazobactam, with bacterial cultures demonstrating Corynebacterium and methicillin-resistant Staphylococcus aureus. Some improvement was noted in the patient's skin after antibiotics were initiated, but he continued to describe worsening "burning and pain" throughout the psoriasis lesions. The patient's care was transferred to the Veterans Affairs hospital where a dermatology inpatient consultation was placed.

Our initial dermatologic examination revealed generalized scaly erythroderma on the neck, trunk, and extremities, sparing the face, palms, and soles (Figure 1).
Multiple crusted and intact vesicles also were present overlying the erythematous plaques on the chest, back, and proximal extremities, most grouped in clusters. The patient endorsed new symptoms of pain and burning. Tzanck smear from the abdomen along with shave biopsies from the left flank and right abdomen were performed, and intravenous acyclovir was initiated immediately after these procedures.

Viral cultures were taken but were incorrectly processed by the laboratory. Tzanck smear showed severe acute inflammation with numerous neutrophils, multinucleated giant cells with viral nuclear changes, and positive immunostaining for HSV and negative immunostaining for herpes zoster. Both pathology specimens revealed an intense acute mixed, mainly neutrophilic, inflammatory infiltrate extending into the deeper dermis as well as distorted and necrotic hair follicles, some of which displayed multinucleated epithelial cells with margination of chromatin that were positive for both HSV-1 and HSV-2 and negative for herpes zoster (Figure 2). The positivity of both HSV strains might represent co-infection or could be a cross-reaction of antibodies used in immunohistochemistry to the HSV antigens. There was acantholysis surrounding the ulceration and extending through the full thickness of the epidermis with a
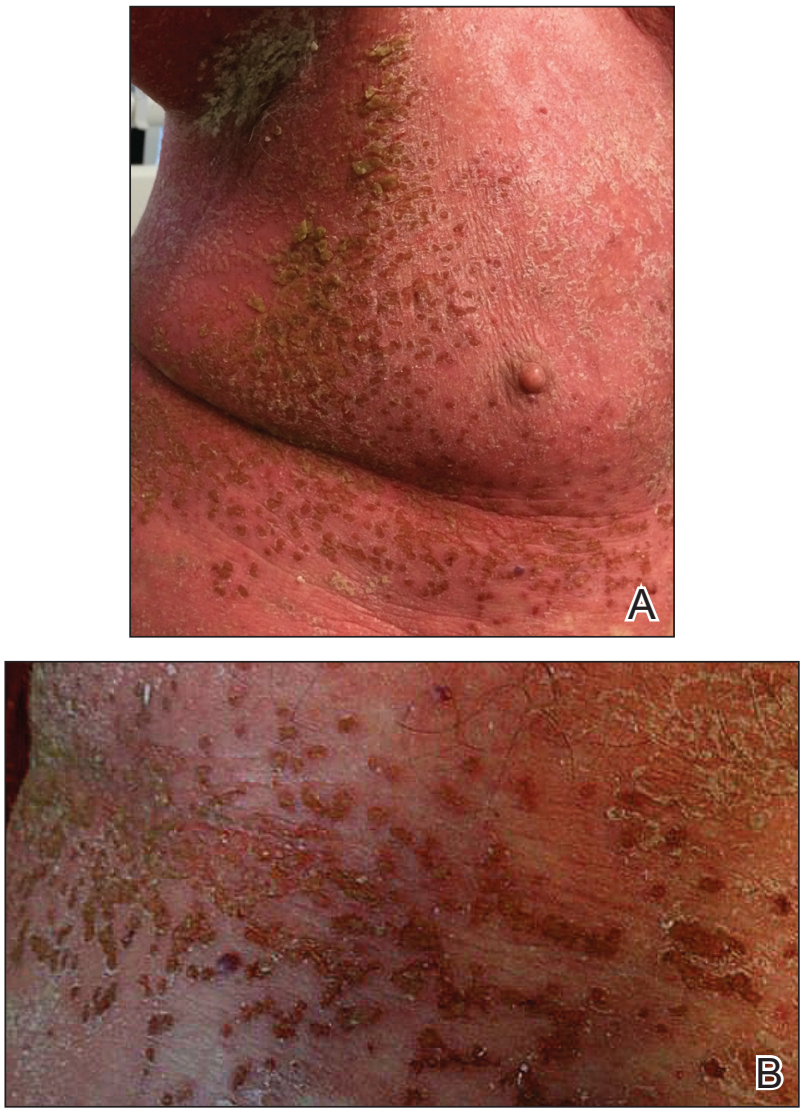

FIGURE 1. A and B, Grouped crusted vesicles on a background of erythroderma on the trunk. 

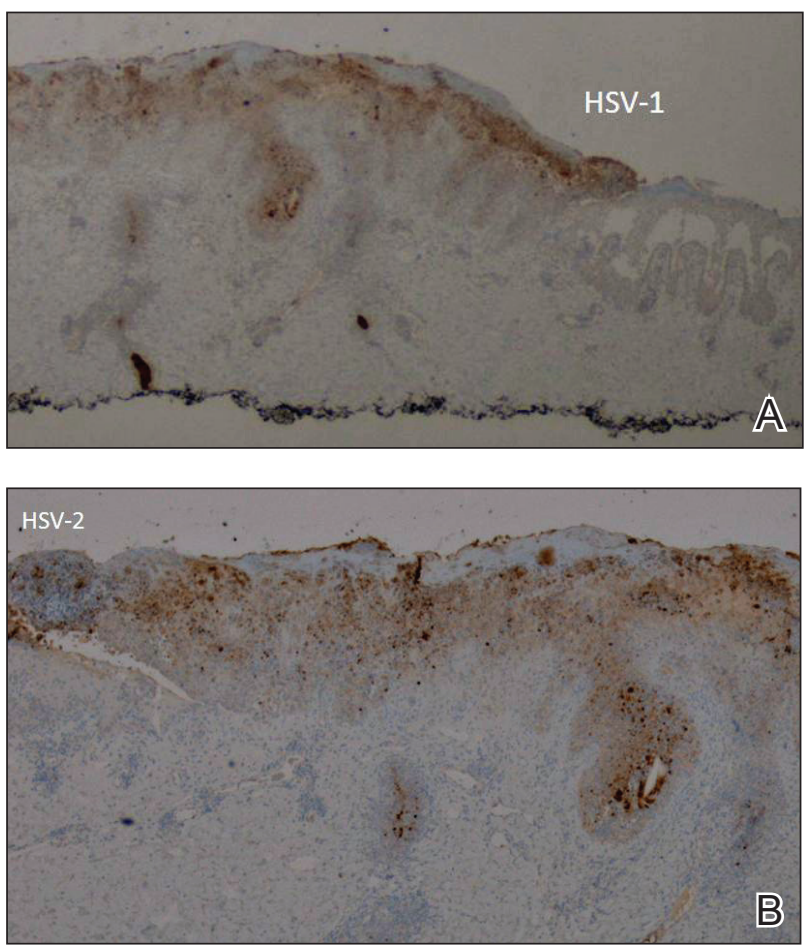

FIGURE 2. A, Positive immunostain for herpes simplex virus type 1 (original magnification $\times 40$ ). B, Positive immunostain for herpes simplex virus type 2 (original magnification $\times 40$ ).

dilapidated brick wall pattern (Figure 3) as well as negative immunohistochemical staining for HSV-1 and HSV-2 antigens. The clinical and histological picture together, along with prior clinical and pathological reports, confirmed the diagnoses of acute erythrodermic HHD with HSV superinfection.

The patient's condition and pain improved within 24 hours on intravenous acyclovir. On the third day, his lesions were resolving and symptoms improved, so he was transitioned to oral acyclovir and discharged from the hospital. Follow-up in the dermatology outpatient clinic 1 week later revealed that all vesicles and papules had cleared, but the patient was still erythrodermic. Because HHD cannot always be distinguished histologically from other forms of pemphigus but yields a negative immunofluorescence, direct immunofluorescence and indirect immunofluorescence were obtained upon patient followup in the clinic and were both negative. Hepatitis $C$ viral loads were undetectable. Consultations to gastroenterology and oncology teams were placed for consideration of systemic agents, and the patient was initiated on oral acitretin $25 \mathrm{mg}$ daily, along with clobetasol as adjuvant therapy for any residual skin plaques. The laboratory results were closely monitored. Within 4 weeks after starting acitretin, the patient's erythroderma had completely resolved. The patient has remained stable since then, except for one episode of secondary Staphylococcus infection that cleared on oral antibiotics. The patient
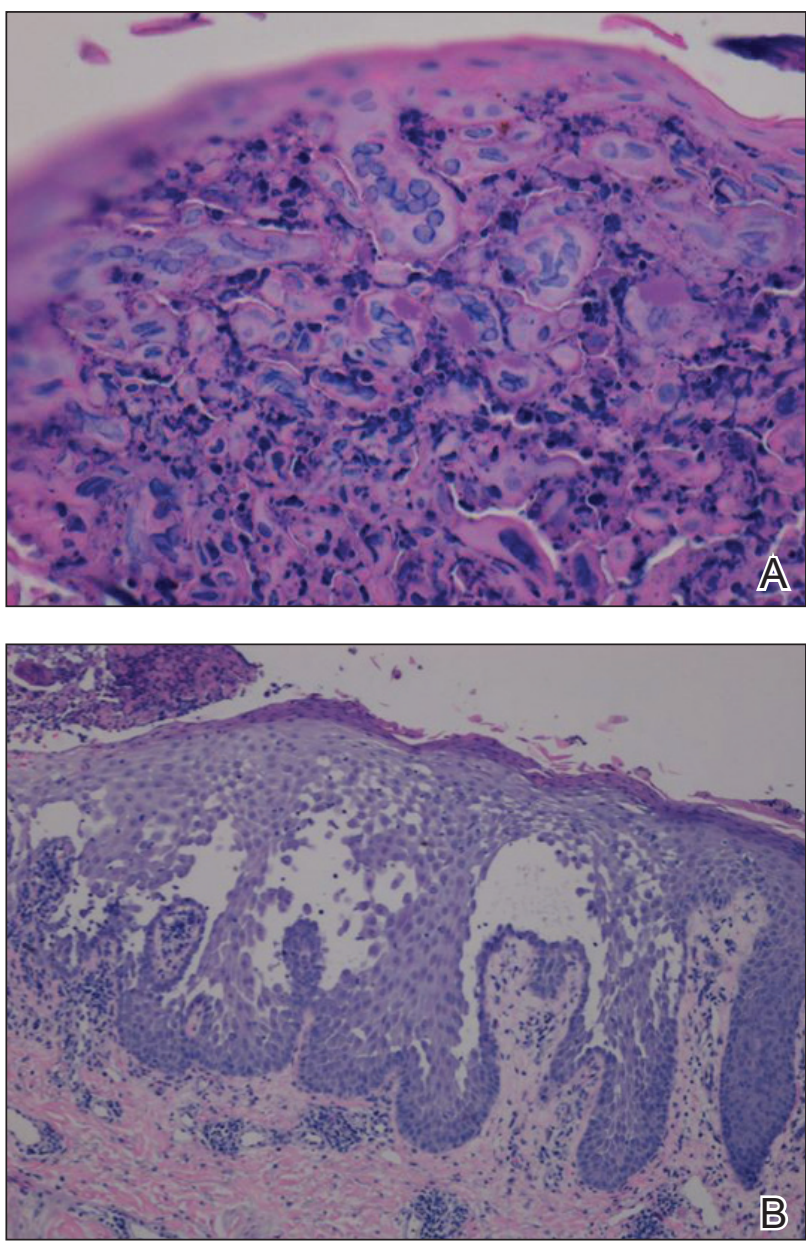

FIGURE 3. A, Multinucleated giant cells in herpes simplex virus infection $(\mathrm{H} \& \mathrm{E}$, original magnification $\times 400)$. B, Acantholysis extending through the full thickness of the epidermis with a dilapidated brick wall pattern $(\mathrm{H} \& \mathrm{E}$, original magnification $\times 40)$.

remains stable and clear on oral acitretin $25 \mathrm{mg}$ daily, with concomitant desonide cream and fluocinonide ointment as needed.

Hailey-Hailey disease is characterized by recurrent episodes of erythema, blisters, and plaques localized to intertriginous and perianal areas. ${ }^{1,2}$ Patients display a spectrum of lesions that vary in severity. ${ }^{8}$ Typical histologic examination reveals a dilapidated brick wall appearance. Pathology of well-developed lesions will show suprabasal acantholysis with minimal dyskeratosis. ${ }^{2}$

The generalized form of HHD is an extremely rare variant of the disease..$^{10}$ Generalized HHD may resemble acute hypersensitivity reaction, erythema multiforme, and toxic epidermal necrolysis. ${ }^{1}$ Chronic diseases, such as psoriasis (as in this patient), also may contribute to a clinically confusing picture. ${ }^{8}$ Hailey-Hailey disease and psoriasis are thought to occasionally koebnerize (isomorphic response) to areas of trauma. ${ }^{16}$ Our patient experienced widespread erythematous papules and plaques not restricted to skin folds. His skin lesions continued to worsen over several 
months progressing to erythroderma. The presence of suprabasal acantholysis in a dilapidated brick wall pattern, along with the patient's history, prior pathology reports, clinical picture, and negative direct immunofluorescence and indirect immunofluorescence studies helped to confirm the diagnosis of erythrodermic HHD.

Hailey-Hailey disease is caused by heterozygous mutations in the ATP2C1 gene on chromosome 3q21-24 coding for a Golgi ATPase called SPCA1 (secretory pathway calcium/manganese-ATPase). ${ }^{9}$ Subsequent disturbances in cytosolic-Golgi calcium concentrations interfere with epidermal keratinocyte adherence resulting in acantholytic disease. Studies of interfamilial and intrafamilial mutations fail to pinpoint a common mutation pattern among patients with generalized phenotypes, ${ }^{9}$ which further supports theories that intrinsic or extrinsic factors such as friction, heat, radiation, contact allergens, and infection affect the severity of HHD disease and not the type of mutation., 3,9

Generalization of HHD is likely caused by nonspecific triggers in an already genetically disturbed epidermis. ${ }^{10}$ Interrupted epithelial function exposes skin to infections that exacerbate the underlying disease. Superimposing bacterial infections are commonly reported in HHD. Staphylococcus, Streptococcus, and Candida species colonize the skin and aggravate the disease. ${ }^{11}$ Much less commonly, HSV superinfection can complicate HHD. ${ }^{3-7}$ No data are currently available about the frequency or incidence of Herpesviridae in HHD. ${ }^{7}$ Some studies suggest that UVB light therapy can be an exacerbating factor in DAR and some but not all HHD patients, ${ }^{12,13}$ while other case reports ${ }^{14,15}$ document clinically improved responses using phototherapy for patients with HHD. Clinicians should remain suspicious and evaluate for HSV infection in refractory or sudden exacerbation of HHD. ${ }^{7}$ Furthermore, coexistent psoriasis and HHD also is a rare entity but has been described, ${ }^{8}$ which illustrates the importance of not attributing all skin manifestations to a previously diagnosed disorder but instead keeping an open mind in case new dermatologic conditions present themselves at a later time.

We present a rare case of erythrodermic HHD and coexistent psoriasis with HSV superinfection. We hope to draw awareness to this association of generalized HHD with both HSV and psoriasis to help clinicians make the correct diagnosis promptly in similar cases in the future.

\section{REFERENCES}

1. Chave TA, Milligan A. Acute generalized Hailey-Hailey disease. Clin Exp Dermatol. 2002;27:290-292.

2. Mohr MR, Erdag G, Shada Al, et al. Two patients with Hailey-Hailey disease, multiple primary melanomas, and other cancers. Arch Dermatol. 2011;147:211-215.

3. Lee GM, Kim YM, Lee SY, et al. A case of eczema herpeticum with Hailey-Hailey Disease. Ann Dermatol. 2009;21:311-314.

4. Zaim MT, Bickers DR. Herpes simplex associated with Hailey-Hailey disease. J Am Acad Dermatol. 1987;17:701-702.

5. Peppiatt T, Keefe M, White JE. Hailey-Hailey disease-exacerbation by herpes simplex virus and patch tests. Clin Exp Dermatol. 2006; 17:201-202.

6. Almeida L, Grossman ME. Benign familial pemphigus complicated by herpes simplex virus. Cutis. 1989;44:261-262.

7. Nikkels AF, Delvenne P, Herfs M, et al. Occult herpes simplex virus colonization of bullous dermatitides. Am J Clin Dermatol. 2008;9:163-168.

8. Chao SC, Lee JY, Wu MC, et al. A novel splice mutation in the ATP2C1 gene in a woman with concomitant psoriasis vulgaris and disseminated Hailey-Hailey disease. Int J Dermatol. 2012;51:947-951.

9. Ikeda S, Shigihara T, Mayuzumi N, et al. Mutations of ATP2C1 in Japanese patients with Hailey-Hailey disease: intrafamilial and interfamilial phenotype variations and lack of correlation with mutation patterns. J Invest Dermatol. 2001;117:1654-1656.

10. Marsch W, Stuttgen G. Generalized Hailey-Hailey disease. Br J Dermatol. 1978;99:553-559.

11. Friedman-Birnbaum R, Haim S, Marcus S. Generalized familial benign chronic pemphigus. Dermatologica. 1980;161:112-115.

12. Richard G, Linse R, Harth W. Hailey-Hailey disease. early detection of heterozygotes by an ultraviolet provocation tests-clinical relevance of the method. Hautarzt. 1993;44:376-379.

13. Mayuzumi N, Ikeda S, Kawada H, et al. Effects of ultraviolet B irradiation, proinflammatory cytokines and raised extracellular calcium concentration on the expression of ATP2A2 and ATP2C1. Br J Dermatol. 2005;152:697-701.

14. Vanderbeck KA, Giroux L, Murugan NJ, et al. Combined therapeutic use of oral alitretinoin and narrowband ultraviolet-B therapy in the treatment of Hailey-Hailey disease. Dermatol Rep. 2014;6:5604.

15. Mizuno K, Hamada T, Hasimoto T, et al. Successful treatment with narrow-band UVB therapy for a case of generalized Hailey-Hailey disease with a novel splice-site mutation in ATP2C1 gene. Dermatol Ther. 2014;27:233-235.

16. Thappa DM. The isomorphic phenomenon of Koebner. Indian J Dermatol Venereol Leprol. 2004;70:187-189. 